\title{
The Characteristics of Chinese Thinking Mode and Its Influence on Language
}

\author{
Yuling Geng \\ College of Languages \\ Xinjiang Normal University \\ Urumqi, China 830017
}

\begin{abstract}
Language determines the way of thinking, and the way of thinking reacts to the language structure. From the perspective of cultural linguistics, the article attempts to reveal that the spiritual culture determines the language psychology, the language psychology determines the way of thinking, and the way of thinking influences the linguistic structure, a language ecological chain, in order to explore the relationship between language and thinking.
\end{abstract}

Keywords—thinking mode; language structure; influence

\section{INTRODUCTION}

The way of thinking is a bridge to communicate culture and language. On the one hand, the way of thinking is closely related to culture, which is a concentrated embodiment of cultural and psychological characteristics, and also has a restrictive effect on various elements of culture. On the other hand, the way of thinking is closely related to language and is the deep mechanism of language generation and development. Language also promotes the formation and development of thinking. The mode of thinking is the integration and unity of the formative thinking, the way of thinking, and the thinking process of the subject in reflecting the object's thinking process. [1]As a typical paradigm-type language, the characteristics of Chinese thinking style have a profound influence on Chinese pronunciation, vocabulary, grammar, semantics and pragmatics.

\section{THE Characteristics OF A CHINESE MODE OF THINKING}

\section{A. The Overall Concept and General Connection}

The thought of "Yin and Yang Five Elements" and "Heaven and Man Oneness" in Chinese philosophy has been deeply accumulated in the cultural and psychological structure of Chinese, forming a powerful collective unconscious. [2]Affected by the yin and yang ideology in Laozi and Zhuang philosophy, Chinese tend to view the world as a yin and yang opposition, thinking that everything in the world has duality. The unity of oppositeness of yin and yang gave birth to Chinese holistic thinking method. "The holistic thinking comprehensively considers the organic connection between heaven, earth, human and nature, society, and life in the relationship network, and pays attention to the overall relevance. Instead of decomposing the whole into parts and analyzing the research one by one; pay attention to the structure and function, not the entities and elements; pay attention to the dialectical method to understand the harmony of diversity and the unity of opposites". For example, people are divided into men and women, things are divided, things are important.

\section{B. Regardless of the Subject and the Guest and the "Master" Set "off"}

Chinese are accustomed to quantifying things from the subject's feelings. This is a cognitive mode in which the subject and the object are separated. It is the observer as the "master" and the observer as the "guest" who participates in the observed things. [3]It mainly considers the observer's perception and perception of the object of observation. In other words, the object is read, copied, abstracted, distinguished, and summarized by the brain. For example, in the case of Zou Ji's "My Country and the North City of Xugong", his wife did not observe Xu Gong, but he only felt love for her husband and he thought subjectively that "Junmeishen, Xu Gonggong and Gonggong also!". It is precisely because of this fact that she disregards the fact that she is "beautiful in the north of the city and the beauty of Qi", and uses her own subjective feelings to judge "comparable beauty". Similarly, in terms of the concept of things, we consider both intuitive and indirect cognition. For example, the "woodpecker" is a kind of bird in terms of intuitive feeling; but indirectly, it is also through eating trees and eliminating pests.

\section{Emphasis on Function and Focus on Perception}

The traditional way of thinking of Chinese is intuitive thinking. Its reasoning is carried out by using the personal experience in life, the direct experience of practice, and the traditional concept of habit and it is carried out by the external relations of things. The basis of this reasoning is to pay attention to the function and the emphasis on perception, paying attention to the function of things as well as the direct feeling of things. [4]"The Analects of Confucius," said: The position is known as a courtesy, and it is well known to be honored. The granary is enriched, food, clothing and food are warm, and the concept of honor and disgrace is well-founded. People can also spontaneously, consciously, and universally pay attention to courtesy and admiration. Book 6: "Those 
who are good-natured, thirsty and drink first, drink too much, and most of them lose their breath. When thirst is hurt, blood is eaten first but food is full, fullness is hurt, and hunger is hurting the stomach.": "Evening food in the middle of the night should be abstained. It is advisable to apply for a late night snack in Shenxi." The above two paragraphs reflect the ancient people's superficial experience and deep understanding of food and clothing.

\section{Depicting the Image and Righteousness}

"The Analects of Confucius • Zi Lu": Zi Lulu said: "The guardian will be the ruler, and the son will be the first?" The child said: "Must also be named!" Zilu said: "There is nothing but the son's wife! Is it right?" The child said: "Yeji, from also! The gentleman knows nothing about him, and the cover is as good as it is. If the name is not correct, then the words are not correct; if the words are not correct, the matter is not completed; if the matter is not completed, the ritual is not happily; the penalties are not in the penalty; if the penalty is not in the penalty, then the people have nothing to do with it. [5]Therefore, the name of the gentleman must be able to speak and the words must be workable. The gentleman speaks in his words and nothing is wrong. A traditional way of thinking of the nation: justified, that is doing something in the name of justification, the truth also makes sense. In the social life of Chinese, everything must be presented with facts, principles, and even rebellion. It is also necessary to compile a statement of "Great Chu Xing and Chen Sheng Wang",

\section{E. Scatter Perspectives and Eclectic}

Scatter perspective is also called multi-point perspective, that is, different objects have different vanishing points. This perspective method is more common in Chinese painting. Scatter perspective method is not rigidly adhered to a point of view. It is multi-viewpoint. When expressing a scene, it can use the multi-viewpoints to deal with similarly-sized scenes that are near-big and small. Scatter perspective method can fully express all aspects of scenery with large space span. This is a great advantage of traditional Chinese painting. As a tradition of Chinese art, the perspective of scatter has also become a way of thinking of Chinese, that is, thinking from multiple perspectives, at multiple levels, and in multiple forms, and avoiding dull, single thinking patterns. Taking the self-rationality of Chinese as an example, the purpose is mainly to respect others' humility and respect, to consider both their own feelings and the sake of the other side. Under the premise of not being humbled, they should respect each other to the greatest extent and avoid embarrassment. For example, when accepting gifts from others, it is often said "Let you spend money" and "What kind of money do you spend?" The person giving the gifts will also take the opportunity to be polite, like "What to spend" or "Slightly meaningless" and so on.

\section{THE INFLUENCE OF CHINESE MODE OF THINKING ON LANGUAGES}

\section{A. Effect on Speech}

Due to the holistic nature, Chinese sounds, rhymes, and tones constitute whole-syllables, and consonants and vowels are all relative to their overall unit syllables. [6]As for tones, it is the height of the entire syllable. Even each phoneme is a whole, which is the sum of the pronunciation method and the part of the pronunciation. For example, in the syllable "du", since $d$ is a consonant at the beginning of a syllable, it is called an initial; uo is the part behind the initial, called the vowel; the high tone-although it is above the rhyme o, it actually It is the pitch performance of the entire syllable.

\section{B. Influence on the Text}

The development of Chinese characters from pictures and symbolic words into today's unique cubes is not a random result. It is closely related to the practical, multi-imagining, practical and rational thinking that embraces all things of Chinese and the spirit of oneness, oneness, and oneness. Due to the preference for image portrayal, pictograms have become the basis for character creation. The pictograms are like objects, things like humans, and images like devices. The object is the objective existence between heaven and earth. It is the basic condition for people to intervene. Man is the spirit of all things between heaven and earth, and has the wisdom to use and transform everything. The device is the product of man's action in nature and the crystallization of human wisdom. But overall, these three are objective entities. Chinese characters are basically composed of these objective entities.

The same holds true for pictograms and for the word. In the same way, $80 \%$ of Chinese characters are in the form of phonograms because of their overall relevance. The phonograms are divided into sound side and shape side. The former represents pronunciation and the latter represents the classification of meaning. This "characteristic of looking at the word" is precisely the inevitable result of comprehensive consideration and dialectical cognition. Taking "steel" as an example, the sound "oka" means that its pronunciation is "gāng" ; the shape of "gold" means that it belongs to steel. As a result, the meaning of "steel" immediately jumped on paper.

\section{Influence on Vocabulary}

Chinese righteousness and decency require that they must be theoretical when they are named so that they can pass. In the contact between Chinese and other ethnic groups, many foreign words have entered Chinese. However, foreign words in Chinese are not only a way of transliteration, but also transliteration + free translation, transliteration + generics, transliteration + free translation, free translation. From the perspective of the vitality of foreign words in Chinese, the transliteration words are relatively short. For example, the telephone in English, after translating into Chinese, was first transliterated as "telephone", and later it was translated as "telephone", and it has been universal until now. 


\section{Effect on Grammar}

The philosophical thinking of "Heaven and Man Oneness" engenders Chinese holistic thinking and intuitionist thinking characteristics. This holistic and ambiguous way of thinking has also resulted in the paradoxical features of Chinese syntax. The conjunctions and quotations have not been syntactically complete. It can be said that "a person who eats ten meals in a pot" can also say that "ten people eat a pot of rice." It can be said that "Daddy you want to die us" or you can say "Daddy, we want to die you."

Affected by the ambiguous thinking mode, the subjective predicate component in Chinese sentences is incomplete, there are a large number of non-subjective sentences, and there are no obvious passive voice markers. The grammatical structure of Chinese sentences is transformed with the eyes of the speaker. Chicken does not eat." It can express "I don't want to eat chicken" and can also express "chicken does not eat". The former starts from people and the latter starts from chicken. The perspective of the speaker changes freely; things can be dropped on the ground. It can also be dropped to the ground ; it can be said that "a man 30 is a flower", it can be said that "a girl 18 a flower", the farmers often say "a crop of flowers, relying on fat as a family. "What is not clear about the "one flower" here is the embodiment of its overall ambiguity.

\section{E. Impact on Semantics}

The structure of Chinese sentences emphasizes the form of meaninglessness, and comprehends intuitively, which makes the semantic representation often obscured and not as clear as the morphological language. [7]Such as Wen Tingyun's "Shangshan Shangxing" in the famous sentence: chicken sound Mao shop month, the track of frost bridge. The author's use of words is very concise, "chicken voice" do not know is the rooster crowing, or the hen's howling, do not know whether it is a chicken or a few chickens, to correctly understand the meaning of this poem, the reader must from the overall structure Instead of focusing on individual words, the artistic conception of the original text is grasped. Chinese holistic thinking: its language feature is that the boundary between the thinking subject and the thinking object is very vague, emphasizing the way of understanding from the whole to the local and from the inside to the outside, and the Chinese grammar is flexible, re-thinking and expressing bamboo structures, habits rely on the connection of semantics or sentence meanings to reflect the integrity of the language form and the consistency of semantics.

\section{F. Impact on Pragmatics}

Because Chinese image rethinks the image and focuses on the concept, it uses metaphors and symbolic techniques in textual expressions. It likes to use vivid language like utensils and semantically implicit euphemisms. In addition, Chinese use the circular thinking, which is like circling around and gradually reaches a climax. The chapters are naturally arranged according to time and space, and the cultural semantic metaphors of the words focus on metaphorical metaphor. [8]Take Li Bai's "Gift of Wang Lun" as an example: Li Bai would like to go on a boat and suddenly hear the song on the shore. Peach Pond is a thousand feet deeper than Wang Lun sent me. Poetry does not directly show that Wang Lun's deep friendship and friendship with Li Bai, but the use of "Peach Blossom Pond depth of water" to compare, euphemistically stated that the friendship of Wang Lun was even deeper than the water in Taohuatan.

\section{CONCLUSION}

Language is the product of social development and it develops with the development of society. This is the external cause of language evolution. The internal factors that control the evolution of language are not only the unbalanced development of language elements, but also the unique way of thinking of language users. Under the influence of the Chinese thinking mode, Chinese has formed a different spelling in pronunciation, words, vocabulary, grammar, semantics, and pragmatics that is different from the Indo-European language system in terms of overall spelling, literary literacy, righteousness, immutability, and ambiguity, implicit characteristics. Of course, it is foreseeable that with the development of an informationbased society, the way of thinking of Chinese is constantly changing, which is bound to affect the evolution and development of Chinese.

\section{REFERENCES}

[1] Shen Xiaolong.Outline of Cultural Linguistics[M].Guiling:Guangxi Education Press, 1996:168.

[2] Feng Youlan.A Brief History of Chinese Philosophy[M]. Peking:Peking University Press, 2012:74.

[3] Zhang Qiannian.Chinese Humanistic Spirit[M]. Guiyang:Guizhou People's Publishing House, 2017:172.

[4] Huntington .The important role of culture[M].Peking:Xinhua Press, 2010:231.

[5] Ge Benyi.Chinese Lexicology[M].Jinan:Shandong University Press, 2003:163.

[6] Huang Borong.Modern Chinese[M]. Peking:Higher Education Press, 2010:5.

[7] Jia Yande. Chinese Semantics[M].Peking:Peking University Press, 2001:35.

[8] Sun Rujian. Modern Chinese Pragmatics[M]. Wuhan:Huazhong University of Science and Technology Press, 2014: 58. 